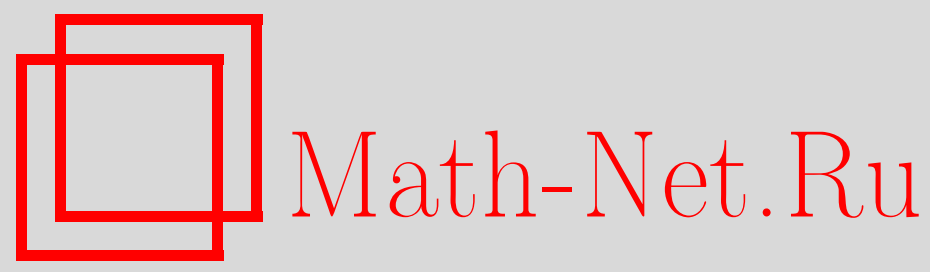

В. С. Самовол, Локальные гладкие преобразования дифференциальных уравнений, сохраняющие линейные автоморфизмы, Матем. заметки, 2002, том 71, выпуск 4, 590-603

DOI: https://doi.org/10.4213/mzm370

Использование Общероссийского математического портала Math-Net.Ru подразумевает, что вы прочитали и согласны с пользовательским соглашением http://www.mathnet.ru/rus/agreement

Параметры загрузки:

IP : 54.174 .149 .18

26 апреля 2023 г., $17: 34: 16$

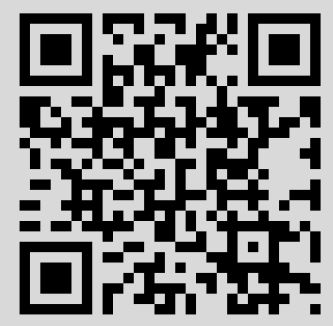




\title{
ЛОКАЛЬНЫЕ ГЛАДКИЕ ПРЕОБРАЗОВАНИЯ ДИФФЕРЕНЦИАЛЬНЫХ УРАВНЕНИЙ, СОХРАНЯЮЩИЕ ЛИНЕЙНЫЕ АВТОМОРФИЗМЫ
}

\author{
В. С. Самовол
}

\begin{abstract}
Настоящая работа посвящена проблемам, связанным с локально гладкими преобразованиями обыкновенных дифференциальных уравнений, сохраняющими их линейные автоморфизмы.

Библиография: 6 названий.
\end{abstract}

Введение. В данной работе мы продолжаем изучение систем обыкновенных дифференциальных уравнений с линейными автоморфизмами, начатое в статьях [1], [2]. Напомним, что речь идет о таких системах, правая часть которых не меняется в результате некоторой линейной замены переменных [3, гл. 3, п. 1.10].

Одним из наиболее эффективных методов анализа дифференциальных уравнений является их упрощение путем подходящего преобразования координат. В этой связи важным является вопрос о сохранении имеющегося автоморфизма после таких преобразований. Для формальных и аналитических систем этот вопрос исследован в [3]. В данной работе эта задача изучается для гладких систем в окрестности невырожденной особой точки.

Рассматривается вещественная система обыкновенных дифференциальных уравнений

$$
\dot{\xi}=\frac{d \xi}{d t}=Q(\xi)
$$

где $\xi, Q(\xi)$ - векторы из $\mathbb{R}^{r}, Q(\xi)$ - функция класса $C^{l}$ в некоторой окрестности нуля (здесь $l>0$ - целое число или $\infty$ ), $Q(0)=0$, матрица $\widetilde{A}=Q^{\prime}(0)$ не имеет собственных чисел с нулевой действительной частью. Точка $\xi=0$ является неподвижной точкой для системы (1).

Пусть при линейном невырожденном преобразовании

$$
\xi=B \eta, \quad t=\mu \tau
$$

система (1) переходит в систему

$$
\dot{\eta}=\frac{d \eta}{d \tau}=\widetilde{Q}(\eta)
$$

Работа выполнена при финансовой поддержке Российского фонда фундаментальных исследований, грант № 00-01-00177. 
где

$$
\widetilde{Q}(\eta)=\mu B^{-1} Q(B \eta) .
$$

Если при этом оказьвается, что $\widetilde{Q}(\eta) \equiv Q(\eta)$, то преобразование $(2)$ называется $л и$ нейным автоморфизмом системы (1) (см. [3]). В дальнейшем предполагается наличие у системы (1) линейного автоморфизма (2). Ниже для краткости слово линейньй мы будем часто опускать. Матрицу $B$ будем назьвать матриией автоморфизма.

Нас прежде всего будет интересовать возможность сохранения автоморфизма (2) системы (1) при ее приведении к нормальной форме (гладкая нормализация), а также при приведении системы к линейному виду (гладкая линеаризация). В работах [1], [2] было показано, что для сохранения автоморфизма (2) при указанных преобразованиях системы (1) достаточно, чтобы собственные числа матрицы $B$ автоморфизма являлись корнями некоторой целой степени из единицы, а жорданова форма этой матрицы была диагональной матрицей.

В данной работе мы покажем, что для сохранения автоморфизма при гладкой нормализации и гладкой линеаризации системы достаточно, чтобы спектр матрицы $B$ автоморфизма (2) находился на единичной окружности, а жорданова форма этой матрищы была диагональной матрицей. Это условие мы назовем условием ортогональности, так как оно означает, что матрища $B$ вещественно-подобна ортогональной матрице.

В работах [1], [2] были приведены примеры, показьвающие существенность расположения спектра матрицы автоморфизма на единичной окружности. В данной статье будет построен пример, показывающий существенность условия диагональности жордановой формы матрищы автоморфизма.

1. Структура автоморфизма. Из равенства (4), в частности, следует, что $\widetilde{A}=$ $\mu B^{-1} \widetilde{A} B$, где $\widetilde{A}=Q^{\prime}(0)$. Приравнивая определители правой и левой частей данного равенства, получаем для вещественной системы (1) и вещественного преобразования (2), что $\mu= \pm 1$.

Отметим, что если $\xi\left(\xi^{0}, t\right)$ - решение системы $(1)$, удовлетворяющееначальному условию $\xi\left(\xi^{0}, 0\right)=\xi^{0}$, и $(2)$ - автоморфизм этой системы, то имеют место равенства

$$
\xi\left(B \xi^{0}, t\right)=B \xi\left(\xi^{0}, \mu t\right), \quad \xi\left(B^{-1} \xi^{0}, t\right)=B^{-1} \xi\left(\xi^{0}, \mu t\right) .
$$

Действительно, поскольку (2) является автоморфизмом системы $(1)$, то вместе с $\xi\left(\xi^{0}\right.$, $t)$ решением этой системы является и функция $\xi_{1}\left(\xi^{0}, t\right)=B^{-1} \xi\left(B \xi^{0}, \mu t\right)$, причем $\xi_{1}\left(\xi^{0}\right.$, $0)=\xi^{0}$. Из этого и из теоремы единственности, очевидно, следуют равенства (5). Таким образом, оператор сдвига по траекториям системы обладает тем же автоморфизмом, что и сама система.

После соответствующего стандартного линейного преобразования нашу систему можно привести к виду, где матрица $\widetilde{A}=Q^{\prime}(0)$ будет иметь блочно-диагональную форму $\widetilde{A}=\operatorname{diag}\left[A_{1}, A_{2}\right]$, где $A_{1}, A_{2}$ - матрищы, имеющие комплексную жорданову форму, причем спектры этих матриц расположены соответственно слева и справа от мнимой оси. Не ограничивая общности, будем считать, что система (1) уже имеет такой вид. При этом, разумеется, надо иметь в виду, что матрица автоморфизма также подвергается соответствующему преобразованию. Будем считать, что матрица $B$ уже является преобразованной матрищей автоморфизма. Перепишем систему (1) более подробно:

$$
\dot{x}^{1}=A_{1} x^{1}+f_{1}\left(x^{1}, x^{2}\right), \quad \dot{x}^{2}=A_{2} x^{2}+f_{2}\left(x^{1}, x^{2}\right) .
$$


Здесь и далее необходимо иметь в виду, что система (6) уже не является вещественной. Однако поскольку исходная система (1) вещественна, то в системе (6) (а также при всех ее дальнейших преобразованиях) будет соблюдаться так называемое условие вещественности (см. [3, гл. 3 , п. 1.9]). Этому же условию удовлетворяет и матрища $B$ автоморфизма системы (6).

Рассматривая равенство $\widetilde{A}=\mu B^{-1} \widetilde{A} B$ как матричное уравнение относительно невырожденной матрищы $B$ (см. [4, с. 199-203]) и учитывая блочно-диагональную форму матрицы $\widetilde{A}$, приходим к следующим выводам.

1. Если $\mu=1$, то матрица $B$ имеет блочно-диагональную структуру $B=\operatorname{diag}\left[B_{1}, B_{2}\right]$ и справедливы равенства

$$
\begin{gathered}
A_{1} B_{1}=B_{1} A_{1}, \quad A_{2} B_{2}=B_{2} A_{2}, \\
f_{1}\left(B_{1} x^{1}, B_{2} x^{2}\right)=B_{1} f_{1}\left(x^{1}, x^{2}\right), \quad f_{2}\left(B_{1} x^{1}, B_{2} x^{2}\right)=B_{2} f_{2}\left(x^{1}, x^{2}\right) .
\end{gathered}
$$

2. Если $\mu=-1$ (это соответствует так называемой обратимой системе), то матрицы $A_{1}$ и $A_{2}$ удовлетворяют равенству $A_{1}=-A_{2}$. Ниже в этом случае будем обозначать $-A_{1}=A_{2}=A$. Таким образом, жорданова форма матрицы $\widetilde{A}$ имеет вид $\operatorname{diag}[-A, A]$, где спектр матрицы $A$ расположен справа от мнимой оси. Размер матрицы $A$ будем обозначать буквой $n$. Из [4] также следует, что матрица $B$ имеет вид

$$
B=\left(\begin{array}{cc}
0 & B_{1} \\
B_{2} & 0
\end{array}\right)
$$

где $B_{1}, B_{2}-$ квадратные матришы, размеры которых равны $n$. Также справедливы равенства

$$
\begin{array}{cl}
A B_{1}=B_{1} A, & A B_{2}=B_{2} A \\
f_{1}\left(B_{1} x^{2}, B_{2} x^{1}\right)=-B_{1} f_{2}\left(x^{1}, x^{2}\right), & f_{2}\left(B_{1} x^{2}, B_{2} x^{1}\right)=-B_{2} f_{1}\left(x^{1}, x^{2}\right) .
\end{array}
$$

В дальнейшем мы будем рассматривать только близкие к тождественным преобразования системы (6). При этом задача состоит в том, чтобы при таких преобразованиях система сохраняла автоморфизм (2).

2. “Выпрямление” устойчивого и неустойчивого многообразий. Хорошо известно, что у системы (6) имеются инвариантные многообразия: устойчивое $\left(x^{2}=\right.$ $\left.\varphi\left(x^{1}\right)\right)$ и неустойчивое $\left(x^{1}=\psi\left(x^{2}\right)\right)$. Широко распространенным и весьма полезньп приемом является “вьпрямление" этих многообразий, т.е. такая замена переменных, после которой они преврашаются в координатные подпространства. В нашем случае необходимо позаботиться о том, чтобы при такой замене переменных сохранялся автоморфизм системы. Сформулируем соответствующее утверждение, доказательство которого дано в [1] (случай $\mu=1$ ) и в [2] (случай $\mu=-1$ ).

Рассмотрим вектор

$$
H(z)=\left(\begin{array}{l}
\psi\left(x^{2}\right) \\
\varphi\left(x^{1}\right)
\end{array}\right), \quad z=\left(\begin{array}{l}
x^{1} \\
x^{2}
\end{array}\right)
$$


Tеорема 1. Для вектора $H(z)$ справедливо равенство $H(B z)=B H(z)$.

Утверждение теоремы по сути дела означает, что "выпрямляющая" замена переменных

$$
y^{1}=x^{1}-\psi\left(x^{2}\right), \quad y^{2}=x^{2}-\varphi\left(x^{1}\right)
$$

коммутирует с автоморфизмом системы и, следовательно, преобразованная система будет, как и исходная, обладать автоморфизмом (2), а устойчивое и неустойчивое многообразия преобразований системы будут являться линейными подпространствами $y^{1}=0$, $y^{2}=0$.

\section{3. Локальная гладкая эквивалентность и нормализация систем уравне-}

ний. Следующий вопрос, которьй нас интересует, заключается в анализе ситуации, описьваемой известной теоремой Стернберга-Ченя о локальной гладкой эквивалентности систем обыкновенных дифференциальных уравнений (см. [5, теорема 12.2]). Достаточные условия такой эквивалентности и методы построения сопрягающего диффеоморфизма хорошо известны. Остается выяснить возможность сохранения автоморфизма системы уравнений при соответствующих ее преобразованиях.

Рассмотрим две системы уравнений

$$
\begin{aligned}
& \dot{\xi}=Q_{1}(\xi), \\
& \dot{\eta}=Q_{2}(\eta),
\end{aligned}
$$

где $\xi, \eta, Q_{1}, Q_{2} \in \mathbb{R}^{r}, r=n_{1}+n_{2}$, функции $Q_{1}, Q_{2}$ принадлежат классу $C^{l}, l>0$ - целое число или $\infty, Q_{1}(0)=Q_{2}(0)=0$,

$$
\frac{\partial Q_{1}(0)}{\partial \xi}=\frac{\partial Q_{2}(0)}{\partial \eta}=\widetilde{A}=\operatorname{diag}\left[A_{1}, A_{2}\right]
$$

$A_{i}$ - квадратная матрица размера $n_{i}, i=1,2, n_{1}+n_{2}=r$, спектры матриц $A_{1}$ и $A_{2}$ лежат соответственно левее и правее мнимой оси.

Пусть, кроме того, системы (7) и (8) обладают автоморфизмом (2), т.е.

$$
\mu B^{-1} Q_{1}(B \xi) \equiv Q_{1}(\xi), \quad \mu B^{-1} Q_{2}(B \eta) \equiv Q_{2}(\eta)
$$

В соответствии с теоремой 1 будем считать, что у систем (7) и (8) имеются "выпрямленные" инвариантные многообразия

$$
\begin{array}{ll}
M_{1 \xi}=\left\{\xi_{i}=0,1 \leqslant i \leqslant n_{1}\right\}, & M_{2 \xi}=\left\{\xi_{i}=0, n_{1}+1 \leqslant i \leqslant r\right\} \\
M_{1 \eta}=\left\{\eta_{i}=0,1 \leqslant i \leqslant n_{1}\right\}, & M_{2 \eta}=\left\{\eta_{i}=0, n_{1}+1 \leqslant i \leqslant r\right\} .
\end{array}
$$

Через $\Lambda=\left\{\lambda_{i}, 1 \leqslant i \leqslant r\right\}$ будем обозначать спектр матрицы $\widetilde{A}$.

Если существует невырожденный диффеоморфизм $\xi=g(\eta)$ класса $C^{k}, k \geqslant 1$, приводящий систему (7) к виду (8) в некоторой окрестности начала координат, то говорят, что системы (7) и (8) $C^{k}$-эквивалентны (локально әквивалентны). Если при этом система (8) линейна, то говорят о $C^{k}$-линеаризаиии системы (7). Нас интересует существование указанного диффеоморфизма, коммутирующего с линейным автоморфизмом системы, т.е. такого, что $g(B \eta) \equiv B g(\eta)$. В этом случае мы будем говорить о $C^{k}$-эквивалентности систем (7) и (8) с сохранением автоморфизма (2). 
В работах [1], [2] рассматривались причины, вследствие которых с точки зрения вопросов локальной гладкой эквивалентности естественным для автоморфизма системы является условие

$$
B^{\widetilde{m}}=E,
$$

где $E$ - единичная матрища, а $\widetilde{m}>0$ - некоторое целое число. Это означает, что собственные числа матрицы $B$ являются корнями степени $\widetilde{m}$ из единицы, а также, что жорданова форма матрищы $B$ является диагональной матрицей. В [1], [2] было доказано, что условие (9) является достаточньм для сохранения автоморфизма системы при ее гладких преобразованиях. Наряду с условием (9) естественно рассмотреть более слабьй его аналог

$$
\left|\beta_{i}\right|=1, \quad 1 \leqslant i \leqslant r,
$$

где $\beta_{1}, \ldots, \beta_{r}-$ собственные числа матрицы $B$. В [1], [2] были построены примеры двух локально гладко эквивалентных систем (1), обладающих одним автоморфизмом (2), для которого невыполнено условие (10), вследствие чего, как было показано, всякоегладкое преобразование одной системы в другую не коммутирует с этим автоморфизмом.

В [2] был поставлен вопрос о достаточности условия (10) для гладкой эквивалентности систем с сохранением автоморфизма. В данной работе мы ответим на этот вопрос.

Если собственные числа вещественной матрицы лежат на единичной окружности (т.е. удовлетворяют условию (10)) и комплексная жорданова форма этой матрицы диагональна, то будем говорить, что матрица (и соответствующий ей автоморфизм) удовлетворяет условию ортогональности. Данное условие означает, что вещественная жорданова форма матрицы является ортогональной матрицей или, что то же самое, что матрица вещественно-подобна ортогональной матрице. Ниже мы покажем, что условие ортогональности автоморфизма является определяющим при решении вопросов гладкой эквивалентности и гладкой линеаризации с сохранением автоморфизма.

Выше мы отмечали, что построенные в [1], [2] примеры показывают, что часть условия ортогональности, определяемая равенствами (10), является существенной с точки зрения локальной гладкой эквивалентности систем вида (7) и (8) с сохранением автоморфизма. Приведем пример, показьваюший, что условие диагональности жордановой формы матрищы $B$ также является существенньм в этом смысле.

ПримеР 1. Рассмотрим две системы уравнений в $\mathbb{R}^{4}$ :

$$
\begin{gathered}
\dot{x}=x, \quad \dot{y}=y, \quad \dot{z}=2 z+a x y, \quad \dot{w}=2 w+a y^{2}, \quad a \neq 0, \\
\dot{x}_{1}=x_{1}, \quad \dot{y}_{1}=y_{1}, \quad \dot{z}_{1}=2 z_{1}, \quad \dot{w}_{1}=2 w_{1} .
\end{gathered}
$$

Система (12) является линейной частью системы (11). Согласно [6] (см. также условие $S(k)$, приведенное ниже между формулами (36) и (37)) существует диффеоморфизм класса $C^{1}$, линеаризующий систему (11). Системы (11) и (12) обладают автоморфизмом (2), где $\mu=1$ и

$$
B=\left(\begin{array}{llll}
1 & 1 & 0 & 0 \\
0 & 1 & 0 & 0 \\
0 & 0 & 1 & 1 \\
0 & 0 & 0 & 1
\end{array}\right)
$$

Здесь $\beta_{i}=1,1 \leqslant i \leqslant 4$, т.е. условие (10) выполнено.

Наша цель - показать, что не существует невырожденного диффеоморфизма, коммутирующего с указанным автоморфизмом системы и приводящего систему (11) к линейному виду (12). 
ЗАмЕчАНИЕ 1. В задачах огладкой линеаризации с сохранением автоморфизма можно без ограничения общности рассматривать только преобразования, близкие к тождественным. Действительно, если невырожденньй диффеоморфизм

$$
\xi=C \eta+G(\eta), \quad \operatorname{det} C \neq 0, \quad\|G(\eta)\|=o(\|\eta\|),
$$

коммутирующий с автоморфизмом (2), приводит систему (1) к линейному виду

$$
\dot{\eta}=\widehat{A} \eta
$$

то имеет место равенство $\widehat{A}=C^{-1} \widetilde{A} C$, из чего следует, что линейное преобразование

$$
\eta=C^{-1} \zeta
$$

приводит систему (15) к линейной форме

$$
\dot{\zeta}=\widetilde{A} \zeta
$$

Отметим при этом, что поскольку преобразование (14) коммутирует с автоморфизмом (2) системы (1), то и преобразование (16) также коммутирует с данным автоморфизмом. Близкое к тождественному преобразование

$$
\xi=\zeta+G\left(C^{-1} \zeta\right)
$$

будет являться линеаризующим диффеоморфизмом системы (1) того же класса гладкости, что и преобразование (14). Вместе с тем, если преобразование (14) коммутирует с автоморфизмом системы (1), то и преобразование (17) будет также коммутировать с автоморфизмом системы. Это следует из того, что (17) является суперпозицией преобразований (14) и (16), каждое из которых коммутирует с автоморфизмом системы (1).

Перейдем теперь к рассмотрению системы (11). Если бы для системы (11) существовал линеаризующий невырожденньй диффеоморфизм, сохраняющий автоморфизм системы, то согласно замечанию 1 существовал бы и близкий к тождественному диффеоморфизм, коммутирующий с ее автоморфизмом, который мы обозначим через

$$
\eta=\xi+H(\xi), \quad H(\xi) \in C^{1}, \quad\|H(\xi)\|=o(\|\xi\|), \quad \xi=(x, y, z, w), \quad \eta=\left(x_{1}, y_{1}, z_{1}, w_{1}\right)
$$

Поскольку у системы (12) многообразие $L=\left\{z_{1}=0, w_{1}=0\right\}$ инвариантно относительно действия указанного автоморфизма, то и многообразие $L_{1}$ системы $(11)$, являющееся прообразом $L$, также инвариантно относительно данного автоморфизма. Вместе с тем очевидно, что указанные многообразия $L$ и $L_{1}$ являются инвариантными многообразиями систем (12) и (11) соответственно.

Очевидно, $L_{1}$ имеет вид $L_{1}=\left\{z+h_{1}(x, y, z, w)=0, w+h_{2}(x, y, z, w)=0\right\}$, где $h_{i}(\xi) \in C^{1},\left\|h_{i}(\xi)\right\|=o(\|\xi\|), i=1,2$. Разрешая данные уравнения относительно переменных $z, w$, получаем, что при наших предположениях у системы (11) в некоторой окрестности начала координат существует гладкое (класса $C^{1}$ ) инвариантное многообразие вида

$$
z=f(x, y), \quad w=g(x, y)
$$


инвариантное также относительно автоморфизма системы, т.е. выполняется условие

$$
F(B \xi)=B F(\xi)
$$

где

$$
F(\xi)=\left(\begin{array}{c}
0 \\
0 \\
z-f(x, y) \\
w-g(x, y)
\end{array}\right), \quad \xi=\left(\begin{array}{c}
x \\
y \\
z \\
w
\end{array}\right)
$$

Покажем, что это невозможно.

Прежде всего отметим, что условие (19) можно записать следующим образом:

$$
f(x+y, y)=f(x, y)+g(x, y), \quad g(x+y, y)=g(x, y) \text {. }
$$

Пусть окрестность нуля, в которой существует инвариантноемногообразие (18), имеет радиус $\varepsilon$. За счет линейной замены переменных $x=c x_{1}, y=c y_{1}$ число $\varepsilon$ можно сделать сколь угодно большим, при этом изменится только коэффициент $а$ в системе (11). Поэтому без ограничения обшности в дальнейшем будем считать, что $\varepsilon=3$.

Наряду с функцией $g(x, y)$ из инвариантного многообразия (18) рассмотрим функцию $u(x, y)=g(x, y)-a y^{2} \ln |y|$. Отметим, что вместе с функцией $g(x, y)$ функция $u(x, y)$ также принадлежит классу $C^{1}$. Из (20) следует, что функции $f(x, y), u(x, y)$ удовлетворяют равенствам

$$
f(x+y, y)=f(x, y)+u(x, y)+a y^{2} \ln |y|, \quad u(x+y, y)=u(x, y)
$$

Функция $u(x, y)$, очевидно, удовлетворяет уравнению в частных производных

$$
\frac{\partial u(x, y)}{\partial x} x+\frac{\partial u(x, y)}{\partial y} y=2 u(x, y),
$$

т.е. является однородной функцией второй степени.

До сих пор функция $g(x, y)$ и вместе с ней и функция $u(x, y)$ были определены в $\varepsilon$-окрестности начала координат. Доопределим функцию $u(x, y)$ по однородности на всем пространстве. Заметим, что так определенная функция $u(x, y)$ будет по-прежнему удовлетворять второму равенству из (21). Действительно, для произвольной точки $(x, y)$ найдется число $\lambda$ такое, что точка $\left(x_{1}, y_{1}\right)=(x / \lambda, y / \lambda)$ принадлежит $\varepsilon$-окрестности начала координат, где вьполняется (21). Тогда

$u(x+y, y)=u\left(\lambda x_{1}+\lambda y_{1}, \lambda y_{1}\right)=\lambda^{2} u\left(x_{1}+y_{1}, y_{1}\right)=\lambda^{2} u\left(x_{1}, y_{1}\right)=u\left(\lambda x_{1}, \lambda y_{1}\right)=u(x, y)$

и требуемое равенство установлено для всех точек пространства.

Вследствие своей однородности функция $u(x, y)$ удовлетворяет равенству

$$
u(x, y)=y^{2} h\left(\frac{x}{y}\right), \quad y \neq 0, \quad h(x)=u(x, 1) .
$$

Из (21) следуют равенства

$$
f(x+k y, y)=f(x, y)+k u(x, y)+k a y^{2} \ln |y|, \quad u(x+k y, y)=u(x, y),
$$


где $k=1,2, \ldots$.

Подставляя (22) в (23), получаем

$$
h(x+k)=h(x), \quad f(x+k y, y)=f(x, y)+k y^{2} h\left(\frac{x}{y}\right)+k a y^{2} \ln |y| .
$$

Положив в (24) $x=1, y=1 / k$, получим

$$
f\left(2, \frac{1}{k}\right)=f\left(1, \frac{1}{k}\right)+\frac{1}{k} h(k)+\frac{a}{k} \ln \left(\frac{1}{k}\right) .
$$

Кроме того, из $(24)$ следует, что $h(k) \equiv h(0)$ при всех $k=1,2, \ldots$ С учетом этого последнее равенство приобретает вид

$$
f\left(2, \frac{1}{k}\right)=f\left(1, \frac{1}{k}\right)+\frac{1}{k} h(0)+\frac{a}{k} \ln \left(\frac{1}{k}\right),
$$

что противоречит при $a \neq 0 C^{1}$-гладкости функции $f(x, y)$ в точке $(2,0)$. Следовательно, для системы (11) не существует линеаризующего диффеоморфизма, сохраняющего ее линейньй автоморфизм с матрицей (13).

Вопрос о локальной гладкой эквивалентности систем (7) и (8) с сохранением их автоморфизма решается следуюшей теоремой.

Теорема 2. Для систем (7) $u(8)$, әде $l=k+1$, обладающих линейным автоморфизмом (2) с матрицей $B$, которая удовлетворяет условию ортогональности, существует целое число $N=N(k, \Lambda)$, обладающее следующим свойством: если при мальх $\xi$ для всех частных производных $G^{(i)}(\xi)$ порядка $i \leqslant k$ разности $G(\xi)=Q_{1}(\xi)-Q_{2}(\xi)$ справедлива оченка $\left\|G^{(i)}(\xi)\right\| \leqslant D\left\|\left(\xi_{1}, \ldots, \xi_{r}\right)\right\|^{N-i}(D-$ некоторая константа), то для системы (7) существует невырохсденная замена переменных класса $C^{k}$

$$
\xi=g(\eta),
$$

удовлетворяющая условию $g(B \eta)=B g(\eta)$ и приводящая эту систему к виду (8), т.е. системы (7) и (8) $C^{k}$-эквивалентны с сохранением автоморфизма.

Для доказательства теоремы нам потребуется следующая лемма.

ЛЕмма 1. Пусть задана квадратная вещественная матрица С размерности р, удовлетворяющая условию ортогональности. Тогда в $\mathbb{R}^{p}$ существует положительно определенная квадратичная форма $h(x), x \in \mathbb{R}^{p}$, такая, что $h(C x)=h(x)$.

ДокАЗАТЕЛьство. Пусть искомая квадратичная форма задается матрицей $T$, т.е. $h(x)=x^{\prime} T x, x^{\prime}=\left(x_{1}, x_{2}, \ldots, x_{p}\right)$. Необходимо найти такую положительно определенную форму, матрища $T$ которой удовлетворяла бы условию

$$
C^{\prime} T C=T
$$

Существует вещественная невырожденная матрица $U$ такая, что $C=U S U^{-1}$, где $S$ - вещественная жорданова форма матрицы $C$, имеющая вследствие условия ортогональности следующий вид:

$$
S=\operatorname{diag}\left[S_{1}, \ldots, S_{k}, S_{k+1}, \ldots, S_{k+m}\right],
$$


где

$$
S_{i}=\left(\begin{array}{cc}
\cos \varphi_{i} & \sin \varphi_{i} \\
-\sin \varphi_{i} & \cos \varphi_{i}
\end{array}\right)
$$

при $1 \leqslant i \leqslant k, S_{i}=\operatorname{diag}( \pm 1, \ldots, \pm 1)$ при $k+1 \leqslant i \leqslant m, p=2 k+m$.

Нетрудно видеть, что условие (26) эквивалентно равенству $S^{\prime} T_{1} S=T_{1}$, где $T_{1}=$ $U^{\prime} T U$.

Учитывая, что $S^{\prime}=S^{-1}$, в качестве матрицы $T_{1}$ можно взять единичную матрицу, и, следовательно, матрица $T=\left(U U^{\prime}\right)^{-1}$ будет удовлетворять равенству (26). Лемма доказана.

Важную роль в решении вопросов гладкой эквивалентности и линеаризации систем дифференциальных уравнений, обладающих линейным автоморфизмом, играют некоторые вспомогательные функции, построение которых описывается в следующей лемме.

Лемма 2. Для системы (6), обладающей автоморфизмом (2), который удовлетворяет условию ортогональности, существуют положительно определенные квадратичные формы $h_{1}(x), h_{2}(y), h(\xi)=h_{1}(x)+h_{2}(y)$, удовлетворяюшие следующим условиям:

$$
\begin{gathered}
h_{1}\left(B_{1} x\right)=h_{1}(x), \quad h_{2}\left(B_{2} y\right)=h_{2}(y) \quad n p u \quad \mu=1, B=\left(\begin{array}{cc}
B_{1} & 0 \\
0 & B_{2}
\end{array}\right), \\
h_{1}\left(B_{1} x\right)=h_{2}(x), \quad h_{2}\left(B_{2} y\right)=h_{1}(y) \quad n p u \quad \mu=-1, B=\left(\begin{array}{cc}
0 & B_{1} \\
B_{2} & 0
\end{array}\right), \\
h(B \xi)=h(\xi) .
\end{gathered}
$$

ДоказАтЕЛьство. Существование квадратичных форм $h_{1}(x), h_{2}(y)$ при $\mu=1$ непосредственно следует из леммы 1 , так как в этом случае вместе с матрищей $B$ условию ортогональности удовлетворяют и матрицы $B_{1}, B_{2}$. Последовательно полагая в лемме $1 C=B_{1}$ и $C=B_{2}$, получаем требуемое условие (27). Рассмотрим теперь случай $\mu=-1$.

Пусть искомые квадратичные формы задаются соответственно матрицами $T_{1}$ и $T_{2}$, т.е. $h_{i}(x)=x^{\prime} T_{i} x, i=1,2$. Тогда для вьполнения утверждения (28) требуется, чтобы матрицы $T_{1}$ и $T_{2}$ удовлетворяли равенствам

$$
\begin{aligned}
& B_{1}^{\prime} T_{1} B_{1}=T_{2}, \\
& B_{2}^{\prime} T_{2} B_{2}=T_{1} .
\end{aligned}
$$

Подставляя (30) в (31), получаем уравнение для матрищы $T_{1}$

$$
\left(B_{1} B_{2}\right)^{\prime} T_{1}\left(B_{1} B_{2}\right)=T_{1}
$$

При этом необходимо, чтобы матрица $T_{1}$, удовлетворяющая уравнению (32), задавала положительно определенную квадратичную форму. Такое решение у уравнения (32) существует, поскольку матрища $B^{2}=\operatorname{diag}\left[B_{1} B_{2}, B_{2} B_{1}\right]$ удовлетворяет условию ортогональности вместе с матрицей $B$ и, таким образом, этому же условию удовлетворяет матрица $B_{1} B_{2}$. Следовательно, уравнение $(32)$ имеет вид $(26)$ и матрица $T_{1}$ существует. Очевидно, что матрица $T_{2}$, определяемая равенством (30), также задает положительно 
определенную квадратичную форму. Отметим в заключение, что условие (29) следует из (27) и (28). Лемма доказана.

Для доказательства теоремы 2 удобно сначала преобразовать системы (7) и (8) так, чтобы их решения были определены при всех $-\infty<t<+\infty$. Для этого надо заменить функции $Q_{i}(\xi), i=1,2$, функциями, определенными при всех $\xi$ и совпадающими с функциями $Q_{i}(\xi), i=1,2$, при малых $\xi$, но равными линейной части $\widetilde{A} \xi$ вне некоторой окрестности начала координат. При этом важно сохранение автоморфизма преобразованных систем уравнений. Указанное преобразование по существу проводится так же, как и в лемме 3.1 в [5, с. 279-281], с тем лиш изменением, что в качестве сглаживающей функции надо взять такую бесконечно дифференцируемую функцию $\phi(\tau), \tau=h(\xi)$, где $h(\xi)$ - функция, определенная в лемме 2 . При этом функция $\phi(\tau)$ выбирается так же, как и функция $\varphi(t)$ в указанной лемме 3.1 в [5], а именно: $\phi(\tau)=1$ при малых значениях аргумента и равна нулю вне некоторой окрестности нуля, а при остальных $\tau$ она принимает значения между 0 и 1 . Преобразование функций $Q_{i}(\xi), i=1,2$, получается умножением их нелинейных частей на построенную вьше функцию $\phi(h(\xi))$. Чтобы не усложнять обозначения, будем считать, что системы (7) и (8) уже удовлетворяют указанному свойству. Таким образом, решения систем (7) и (8) являются бесконечно продолжаемыми, и область определения отображений $T^{t}: \xi^{0} \rightarrow \xi\left(\xi^{0}, t\right), T_{1}^{t}: \eta^{0} \rightarrow \eta\left(\eta^{0}, t\right)$ совпадает со всем пространством.

ДОКАЗАТЕЛЬСТво ТЕОРЕМЫ 2. Существует несколько способов построения сопрягающего диффеоморфизма. Для того чтобы такой диффеоморфизм коммутировал с автоморфизмом системы, воспользуемся схемой, предложенной в доказательстве теоремы 12.2 в [5, гл. IX].

Рассмотрим функцию $\varphi(\xi)=\varphi(x, y)=-h_{1}(\xi)+h_{2}(\xi)$, где $h_{1}(\xi)=h_{1}(x), h_{2}(\xi)=h_{2}(y)$ - функции, определенные в лемме 2 .

Следуя обозначениям [5, с. 314], определим множества $K^{0}, K^{+}, K^{-}$следующим образом (несколько иначе, чем в [5]):

$$
K^{0}=\{\xi: \varphi(\xi)=0\}, \quad K^{+}=\{\xi: \varphi(\xi)>0\}, \quad K^{-}=\{\xi: \varphi(\xi)<0\} .
$$

Как и в [5], обозначим через $T$ и $T_{1}$ операторы сдвига на единицу времени по траекториям системы (7) и (8) соответственно. Следуя [5], положим $K^{j}=T^{j} K^{0}, j=0, \pm 1, \ldots$, где $Q^{0}=\left\{\xi: \xi \in K^{+}, T^{-1} \xi \in K^{-}\right\}, Q^{j}=\left\{\xi: T^{-j} \xi \in K^{+}, T^{-j-1} \xi \in K^{-}\right\}$.

Отметим, что существует число $\varepsilon>0$ такое, что верны следующие оценки:

$$
\begin{gathered}
-\left(\alpha_{2}+\varepsilon\right) h_{1}\left(\xi\left(\xi^{0}, t\right)\right)<\frac{d h_{1}\left(\xi\left(\xi^{0}, t\right)\right)}{d t}<-\left(\alpha_{1}-\varepsilon\right) h_{1}\left(\xi\left(\xi^{0}, t\right)\right) \\
\left(\alpha_{1}-\varepsilon\right) h_{2}\left(\xi\left(\xi^{0}, t\right)\right)<\frac{d h_{2}\left(\xi\left(\xi^{0}, t\right)\right)}{d t}<\left(\alpha_{2}+\varepsilon\right) h_{2}\left(\xi\left(\xi^{0}, t\right)\right)
\end{gathered}
$$

где $\xi\left(\xi^{0}, t\right)$ - решение системы $(7)$, удовлетворяющее начальному условию

$$
\begin{gathered}
\xi\left(\xi^{0}, 0\right)=\xi^{0}, \quad \alpha_{1}=\min \left(\left|\operatorname{Re} \lambda_{i}\right|, 1 \leqslant i \leqslant r\right), \\
\alpha_{1}-\varepsilon>0, \quad \alpha_{2}=\max \left(\left|\operatorname{Re} \lambda_{i}\right|, 1 \leqslant i \leqslant r\right),
\end{gathered}
$$

$\lambda_{i}$ - элементы спектра матрищы $\widetilde{A}$. Вследствие указанных оценок все определения являются корректными. 
В качестве сопрягаюшего диффеоморфизма для отображений $T^{t}$ и $T_{1}^{t}$ положим согласно упражнению $14.1\left[5\right.$, с. 324] $R(\xi)=T_{1}^{-t(\xi)} T^{t(\xi)} \xi$, где $t(\xi)$ таково, что $T^{t(\xi)} \xi \in K^{0}$, $\xi \notin M_{1 \xi} \cup M_{2 \xi}$. Все рассуждения [5, с. 313-324] полностью сохраняются. Остается убедиться в том, что сопрягающий диффеоморфизм коммутирует с автоморфизмом системы $(7)$.

Из определения функции $\varphi(\xi)$ и равенств $(27),(28)$ следует, что $\varphi(B \xi)=-\varphi(\xi)$, поэтому если $\xi \in K^{0}$, то $B \xi \in K^{0}$. Таким образом, поскольку по определению числа $t\left(\xi^{0}\right)$ $\xi\left(\xi^{0}, t\left(\xi^{0}\right)\right) \in K^{0}$, то $B \xi\left(\xi^{0}, t\left(\xi^{0}\right)\right) \in K^{0}$, откуда, в свою очередь, с учетом (5) следует, что $\xi\left(B \xi^{0}, \mu t\left(\xi^{0}\right)\right) \in K^{0}$. Отсюда получаем, что $t\left(B \xi^{0}\right)=\mu t\left(\xi^{0}\right)$. Далее имеем цепочку равенств

$$
\begin{aligned}
R\left(B \xi^{0}\right) & =T_{1}^{-t\left(B \xi^{0}\right)} T^{t\left(B \xi^{0}\right)} B \xi^{0}=T_{1}^{-t\left(B \xi^{0}\right)} T^{\mu t\left(\xi^{0}\right)} B \xi^{0}=T_{1}^{-t\left(B \xi^{0}\right)} B T^{t\left(\xi^{0}\right)} \xi^{0} \\
& =B T_{1}^{-\mu t\left(B \xi^{0}\right)} T^{t\left(\xi^{0}\right)} \xi^{0}=B T_{1}^{-t\left(\xi^{0}\right)} T^{t\left(\xi^{0}\right)} \xi^{0}=B R\left(\xi^{0}\right) .
\end{aligned}
$$

Четвертое равенство в этой цепочке следует из равенства (5), если его записать для решений системы (8): $\eta\left(B \eta^{0}, t\right)=B \eta\left(\eta^{0}, \mu t\right)$, а в пятом равенстве учтено, что $\mu^{2}=1$. Коммутативность сопрягающего диффеоморфизма и автоморфизма системы установлена. Теорема 2 доказана.

Рассмотрим теперь задачу локальной гладкой нормализации системы (1) с сохранением ее автоморфизма (2). Будем рассматривать системы (1) класса $C^{\infty}$. При этом без ограничения общности считаем, что матрица $\widetilde{A}$ линейной части системы имеет нормальную жорданову форму.

Хорошо известно, что с помощью преобразования, близкого к тождественному,

$$
\xi=u+F(u), \quad\|F(u)\|=o(\|u\|),
$$

где $F(u)$ - полином от своих переменных, можно систему (1) привести к виду

$$
\dot{u}=\widetilde{A} u+R_{1}(u)+R_{2}(u) .
$$

Здесь $R_{1}(u)$ - полином, состоящий из нелинейных резонансных членов степени, не превосходящей некоторого целого числа $N$, а для функции $R_{2}(u)$ вьполняется оценка $\left\|R_{2}(u)\right\|=o\left(\|u\|^{N}\right)$ при $\|u\| \rightarrow 0$. При этом число $N$ можно выбрать сколь угодно большим. Кроме того, из [3, гл. 3 , пा. $1.9,1.10]$ следует, что преобразование (33) можно выбрать таким, чтобы оно удовлетворяло условию вешественности, а также коммутировало с автоморфизмом системы, т.е. $F(B u)=B F(u)$. Таким образом, система (34) также обладает автоморфизмом (2), т.е. для функций $R_{1}(x), R_{2}(x)$ выполняются условия

$$
R_{1}(B u)=\mu B R_{1}(u), \quad R_{2}(B u)=\mu B R_{2}(u) .
$$

Пусть теперь задано некоторое целое число $k>0$. Пусть также для системы (34) выполнено неравенство $N>N(k, \Lambda)$, где $N(k, \Lambda)$ - число, существование которого утверждается в теореме 2. Тогда, применяя к системе (34) теорему 2, получаем, что существует близкое к тождественному преобразование класса $C^{k}$, коммутирующее с автоморфизмом системы, приводящее эту систему к полиномиальной нормальной формe

$$
\dot{v}=\widetilde{A} v+R_{1}(v),
$$

обладающей автоморфизмом (2). Отметим, что из доказательства теоремы 2 следует, что указанное преобразование будет удовлетворять условию вещественности. Таким образом, мы доказали следующую теорему. 
Теорема 3. Для любого иелого числа $k>0$ существует близкое $к$ тождественному преобразование класса $C^{k}$, коммутирующее с автоморфизмом (2) и приводящее в некоторой окрестности начала координат систему (1) класса $C^{\infty} \kappa$ полиномиальной нормальной форме (35).

4. Гладкая линеаризация систем уравнений. Исследуем теперь проблему существования гладкого линеаризующего преобразования системы $(1)$ класса $C^{\infty}$, коммутирующего с ее автоморфизмом (2). В соответствии с теоремой 3 будем считать, что система (1) приведена к полиномиальной нормальной форме (35). Задача, таким образом, сводится к линеаризации системы (35).

Для формулировки заключительных результатов запишем систему (35) в более детальном виде

$$
\dot{v}_{i}=\varepsilon_{i} v_{i-1}+\lambda_{i} v_{i}+a_{i}(v), \quad 1 \leqslant i \leqslant r,
$$

$\varepsilon_{i}=0$ или $\varepsilon, \varepsilon>0, a_{i}(v)=\sum_{|s|=2}^{N} a_{s}^{i} v^{s}, s=\left(s_{1}, \ldots, s_{r}\right)$ - неотрицательные целочисленные наборы, $|s|=\sum_{i=1}^{r} s_{i}$. Считаем, что $\operatorname{Re} \lambda_{i+1} \leqslant \operatorname{Re} \lambda_{i}<0$ при $1 \leqslant i \leqslant n_{1}-1$ и $\operatorname{Re} \lambda_{i+1} \geqslant \operatorname{Re} \lambda_{i}>0$ при $n_{1}+1 \leqslant i \leqslant r$. В случае $\mu=-1$ в соответствии с видом матрицы $\widetilde{A}=\operatorname{diag}[-A, A]$ дополнительно считаем, что $r=2 n, n_{1}=n, \lambda_{i+n}=-\lambda_{i}$, $\varepsilon_{i+n}=\varepsilon_{i}$ при $n+1 \leqslant i \leqslant 2 n$.

Для каждого целочисленного набора $s \in W=\bigcup_{i=1}^{r}\left\{s: a_{s}^{i} \neq 0\right\}$ обозначим

$$
\rho_{p, k}(s)=\left\{\begin{array}{l}
\sum_{i=1}^{p} s_{i}\left|\operatorname{Re} \lambda_{i}\right|-k\left|\operatorname{Re} \lambda_{p}\right|, \quad 1 \leqslant p \leqslant n_{1}, \\
\sum_{i=n_{1}+1}^{p} s_{i} \operatorname{Re} \lambda_{i}-k \operatorname{Re} \lambda_{p}, n_{1}+1 \leqslant p \leqslant r,
\end{array}\right.
$$

Следуя терминологии работы [6], будем говорить, что набор s удовлетворяет условию $S(k)$, если выполняется неравенство $\rho_{k}(s)>0$. Если же это неравенство выполняется для каждого набора $s \in W$, то будем говорить, что система (36) удовлетворяет условию $S(k)$.

Рассмотрим вместе с системой (36) соответствующую линейную систему

$$
\dot{z}_{i}=\varepsilon_{i} z_{i-1}+\lambda_{i} z_{i}, \quad 1 \leqslant i \leqslant r
$$

Имеет место следующее

УТВЕРЖДЕНИЕ [6, теорема 3]. Если для системы (36) выполнено условие $S(k)$, то существует близкое к тохдественному преобразование

$$
v_{i}=z_{i}+H_{i}(z), \quad\left|H_{i}(z)\right|=o(\|z\|), \quad 1 \leqslant i \leqslant r
$$

класса $C^{k}$, приводящее систему (36) к виду (37), т.е. системы (36) и (37) $C^{k}$-эквивалентны.

Таким образом, условие $S(k)$ является достаточным условием $C^{k}$-линеаризации системы (36). Вопрос, которьй нам осталось обсудить, заключается в том, можно ли построить преобразование (38) так, чтобы оно коммутировало с автоморфизмом системы (36). Ответ на этот вопрос дает следующая 
ТЕОРемА 4. Если линейный автоморфизм системы (36) удовлетворяет условию ортогональности, то условие $S(k)$ является достаточным условием для существования преобразования (38), приводящего әту систему к линейному виду (37) и коммутирующего с автоморфизмом системы, т.е. система (36) $C^{k}$-линеаризуема с сохранением ее автоморфизма.

ДокАЗАТЕЛЬСТВО. Искомые функции $H_{i}(z)$ должны удовлетворять системе уравнений в частных производных, характеристическая система для которой имеет вид

$$
\begin{aligned}
\dot{z}_{i} & =\varepsilon_{i} z_{i-1}+\lambda_{i} z_{i}, \quad 1 \leqslant i \leqslant r, \\
\dot{H}_{i} & =\varepsilon_{i} H_{i-1}+\lambda_{i} H_{i}+a_{i}(z+H), \quad 1 \leqslant i \leqslant r .
\end{aligned}
$$

Наша цель - показать, что у системы (39) имеется инвариантное многообразие класса $C^{k}$ вида $H=\varphi(z),\|\varphi(z)\|=o(\|z\|)$, удовлетворяющее условию $\varphi(B z)=B \varphi(z)$. Это будет означать, что отображение $v=z+\varphi(z)$ является искомым.

Следуя технологии линеаризации, разработанной в [6], произведем в системе (39) замену переменных $H=g+\Phi(z), \Phi(z) \in C^{k}$, после которой данная система примет вид

$$
\begin{aligned}
\dot{z}_{i} & =\varepsilon_{i} z_{i-1}+\lambda_{i} z_{i}, \\
\dot{g}_{i} & =\varepsilon_{i} g_{i-1}+\lambda_{i} g_{i}+\widetilde{a}_{i}(z, g)+\widetilde{a}_{i}(z), \quad 1 \leqslant i \leqslant r,
\end{aligned}
$$

где функции $\widetilde{a}_{i}(z, g), \widetilde{a}_{i}(z)$ таковы, что $\widetilde{a}_{i}(z, 0) \equiv 0,\left|\widetilde{a}_{i}(z)\right|=o\left(\|z\|^{N}\right)$, причем число $N$ можно сделать сколь угодно большим.

Ниже мы покажем, что функция $\Phi(z)$ может быть построена так, что

$$
\Phi(B z)=B \Phi(z)
$$

Вследствие этого система (40) обладает линейным автоморфизмом (2) с матрищей $B$.

Применяя к системе (40) доказанную вьше теорему 2 , получаем, что существует преобразование $g=g^{1}+\Psi(z),\|\Psi(z)\|=o(\|z\|), \Psi(z) \in C^{k}$, приводящее эту систему к виду

$$
\begin{aligned}
\dot{z}_{i} & =\varepsilon_{i} z_{i-1}+\lambda_{i} z_{i} \\
\dot{g}_{i}^{1} & =\varepsilon_{i} g_{i}^{1}+\lambda_{i} g_{i}^{1}+\widetilde{a}_{i}\left(z, g^{1}\right), \quad 1 \leqslant i \leqslant r .
\end{aligned}
$$

При этом для функции $\Psi(z)$ вьполняется условие $\Psi(B z)=B \Psi(z)$, т.е. преобразование коммутирует с автоморфизмом системы. У системы (42) многообразие $g^{1}=0$ является инвариантным, следовательно, у системы (40) многообразие $g=\Psi(z)$ также инвариантно, из чего следует, что преобразование $H=\varphi(z)=\Phi(z)+\Psi(z) \in C^{k}$ является искомьм и удовлетворяет требуемому условию $\varphi(B z)=B \varphi(z)$.

Нам осталось показать, что функция $\Phi(z)$ может быть построена так, что будет выполнено условие (41).

При построении вектор-функции $\Phi(z)=\left(\Phi_{1}(z), \ldots, \Phi_{r}(z)\right)$ мы будем следовать конструкции, реализованной в [1], [2]; а именно, определим эту функцию следующим образом:

$$
\Phi_{i}(z)=\sum_{|s|=2}^{N} b_{s}^{i} z^{s} T^{\varpi}, \quad 1 \leqslant i \leqslant r
$$


где $z^{s}$ - резонансные наборы, удовлетворяющие условию $S(k), T^{\varpi}=t_{1}^{\varpi 1} \cdots t_{r}^{\varpi_{r}}-$ мономы от переменных $t_{1}(z), \ldots, t_{r}(z)$, причем функции $t_{j}(z), 1 \leqslant j \leqslant r$, таковы, что производная каждой из них в силу линейной системы (37) равна единице.

Сохраняя идею построения функций $t_{j}(z)$, мы внесем в их конструкцию некоторые изменения. Пусть вначале $1 \leqslant j \leqslant n_{1}$. Для каждого числа $1 \leqslant j \leqslant n_{1}$ определим $q(j)$ как максимальное из чисел $1 \leqslant i \leqslant n_{1}$, для которых $\operatorname{Re} \lambda_{i}=\operatorname{Re} \lambda_{j}$. Для $n_{1}+1 \leqslant j \leqslant r$ число $q(j)$ определим как максимальное среди чисел $n_{1}+1 \leqslant i \leqslant r$, для которых $\operatorname{Re} \lambda_{i}=\operatorname{Re} \lambda_{j}$.

$\mathrm{B}$ качестве функций $t_{j}(z)=t_{j}\left(z_{1}, z_{2}, \ldots, z_{r}\right)$ рассмотрим взятое с обратным знаком время, за которое точка

$$
\widetilde{z}= \begin{cases}\left(z_{1}, z_{2}, \ldots, z_{q(j)}, 0, \ldots, 0\right), & 1 \leqslant j \leqslant n_{1} \\ \left(0, \ldots, 0, z_{n_{1}+1}, z_{n_{1}+2}, \ldots, z_{q(j)}, 0, \ldots, 0\right), & n_{1}+1 \leqslant j \leqslant r\end{cases}
$$

достигнет поверхности $h(\widetilde{z})=\varepsilon$, где $h(\widetilde{z})$ - функция, определенная в лемме 2 .

Из построения функции $h(\widetilde{z})$ следует, что для функций $t_{j}(z)$ выполняются условия

1) при $\mu=1 t_{j}(B z)=t_{j}(z), 1 \leqslant j \leqslant r$;

2) при $\mu=-1 t_{j}(B z)=-t_{n+j}(z), 1 \leqslant j \leqslant n$.

Все остальные рассуждения [1], [2], относящиеся к построению функции $\Phi(z)$, полностью сохраняются. Таким образом, существование требуемого многообразия у системы (39) установлено и, следовательно, для системы (36) существует близкое к тождественному преобразование класса $C^{k}$, приводящее ее к линейному виду (37) и коммутирующее с автоморфизмом (2). Теорема доказана.

Автор выражает искреннюю благодарность А. Д. Брюно за полезные обсуждения вопросов, связанных с проблематикой данной работы.

\section{СПИСОК ЦИТИРОВАННОЙ ЛИТЕРАТУРЫ}

[1] Самовол В. С. Гладкая эквивалентность дифференциальных уравнений и линейные автоморфиизмы // Матем. заметки. 1999. Т. 66. № 4. С. 567-578.

[2] Самовол В. С. Гладкая эквивалентность и линеаризация обратимых систем // Матем. заметки. 2001. Т. 70. № 1. С. 96-108.

[3] Брюно А.Д. Локальньй метод нелинейного анализа дифференциальных уравнений. М.: Наука, 1979.

[4] Гантмахер Ф.Р. Теория матриц. М.: Наука, 1967.

[5] Хартман $\Phi$. Обыкновенные дифференциальные уравнения. М.: Мир, 1970.

[6] Самовол В. С. Линеаризация систем дифференциальных уравнений в окрестности инвариантных тороидальных многообразий // Тр. ММО. 1979. Т. 38. С. 187-219.

Государственный университет, Высшая школа экономики (г. Москва)

Поступило E-mail: samovol@cityline.ru 